\section{SELECTIVE LASER MELTING TECHNOLOGY AND INDIVIDUAL TI-6AL-4V IMPLANTS}

medicine. For this purpose, two areas of interest were tested. In the first parts, mechanical properties are studied on the basis of uniaxial tensile test. In the second part, shape accuracy is evaluated on two types of cranial implant. Under the SLM process, the Ti-6Al-4V alloy is known to produce large inner stresses which tend to distort the part. This phenomenon is not acceptable in the case of individual implants because the fit of the part towards the bone tissue has to be precise for successful surgery.

\section{MATERIALS AND METHODS}

Quality of input material in terms of topology and size distribution of powder particles is one of the most important features of the SLM process. First part of the work is therefore dedicated to this topic. Following part describe experimental setup for evaluation of mechanical properties for SLMprocessed Ti-6Al-4V alloy in as-build and heat treated condition. Last part of the work show shape accuracy analysis on individual cranial implant.

\subsection{Powder inspection}

Ti-6Al-4V powder was supplied by SLM Solutions AG (Luebeck, Germany). The sample of the powder was examined via Scanning Electron Microscope (SEM) for evaluation of particle topology (Fig. 1). Particles are spherical with very low amount of irregularities. In the next step, size distribution was evaluated using Laser Diffraction method on CILAS $990 \mathrm{~L}$ particle analyser (Fig. 2). The powder inspection show typical size distribution for SLM process. Majority of particles fall within the range of 15-45 $\mu \mathrm{m}$.

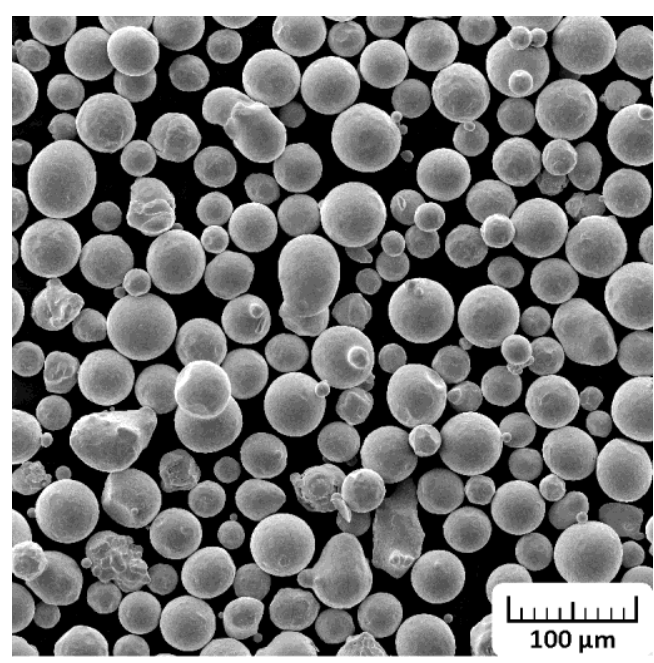

Figure 1. SEM image of Ti-6Al-4V powder implant using various modelling techniques [Eufinger 1995].

One of the most used materials for reconstructive surgery which can be processed using additive technologies is Ti-6Al-4V alloy [Hao 2016]. Given material can be used for production of implants with minimal porosity. Nowadays, the two key technologies for fabrication are Selective Laser Melting (SLM) and Electron Beam Melting (EBM) [Murr 2012]. Although the basic principle of the technologies is the same - layer-wise connection of fine metal powder - the source of heat for powder melting is different. Despite great potential of additive manufacturing of individual implants, there are still many limiting factors which prevent wider application of such an approach.

The aim of this article is to study key properties of final parts fabricated using SLM technology with regard to its final use in 


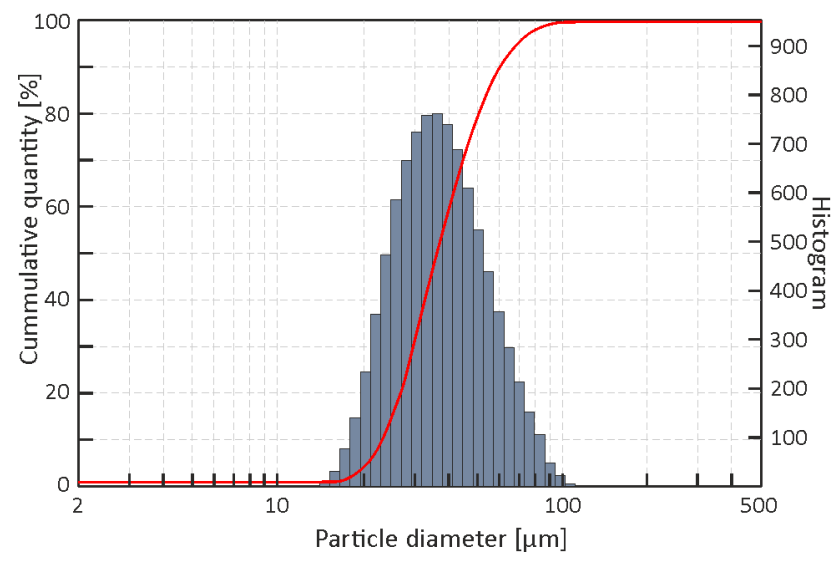

Figure 2. Size distribution analysis of Ti-6Al-4V powder

In the last part of powder inspection, humidity of the powder was verified using B+B Sensors Hytelog RS232 probe. Measured value of $4.2 \%$ r.h. was found suitable for further use of the powder.

\subsection{Tensile test specimens}

Monotonic properties of the material were tested on full scale specimens which shape was derived from ASTM E606 standard (Fig. 3). With regard to a need of machining of the final shape, specimens were fabricated with $0.5 \mathrm{~mm}$ body offset.

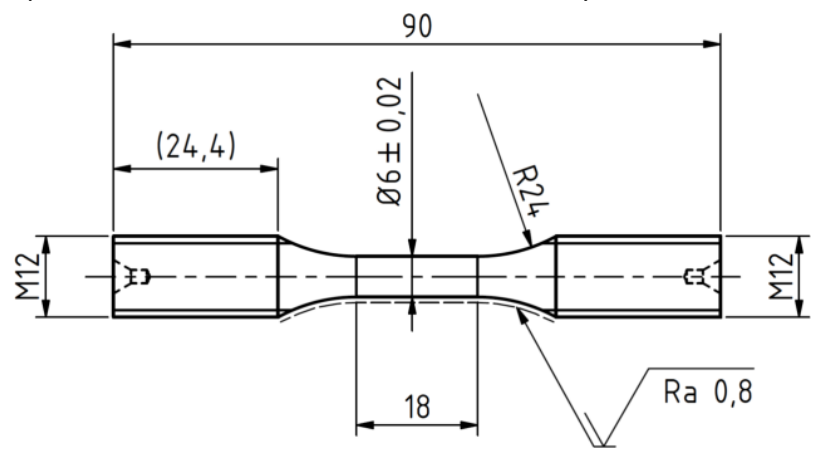

Figure 3. Dimensions of tensile test specimen.

\subsection{Shape accuracy specimens}

For the shape accuracy testing purposes, cranial implant was chosen as a sample part. Cranial operations represent one of the most demanding fields in clinical surgery and thus the implant has to match initial CAD data with minimal deflections. Moreover, parts in the form of large thin sheet are very likely to undergo large deflections after the SLM process.

In order to test capabilities of additive manufacturing, two different versions of the implant were created. In the first case, the geometry was left in condition typical for standard fabrication processes - fully dense plate with no special features. In the second case, lightweight structure was incorporated into the geometry. For SLM process, this step has several advantages - it reduces the energy which is put into individual layers and it also dramatically reduces build time of the part. Due to the low thickness of the implant, no standard lattice structure with thin rods was used. The structure is formed by protrusion of 2D pattern through body of the part. Individual bars which create the structure has rectangle crosssection with dimension of $1,5 \times 2 \mathrm{~mm}$.

Both geometries of the cranial implant were built in the same orientation and using similar support structures. Specifically, combination of hard pins with diameter of $1 \mathrm{~mm}$ and block supports was used (Fig 4).

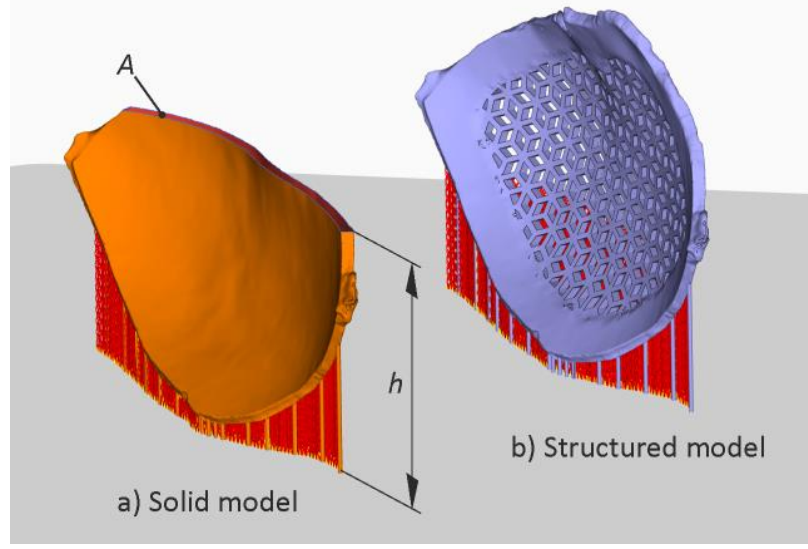

Figure 4. Solid and structured model of cranial implant.

After setting the position for building job, area of the layer for each slice was analysed. Output of this analysis is expressed graphically in the fig. 5 . The graph clearly demonstrates that the surface which is exposed to laser is considerably lower in the case of structured model. Apart from this fact, area of the layer also remains on rather same level of approximately $200 \mathrm{~mm}^{2}$ throughout the structured part of the geometry.

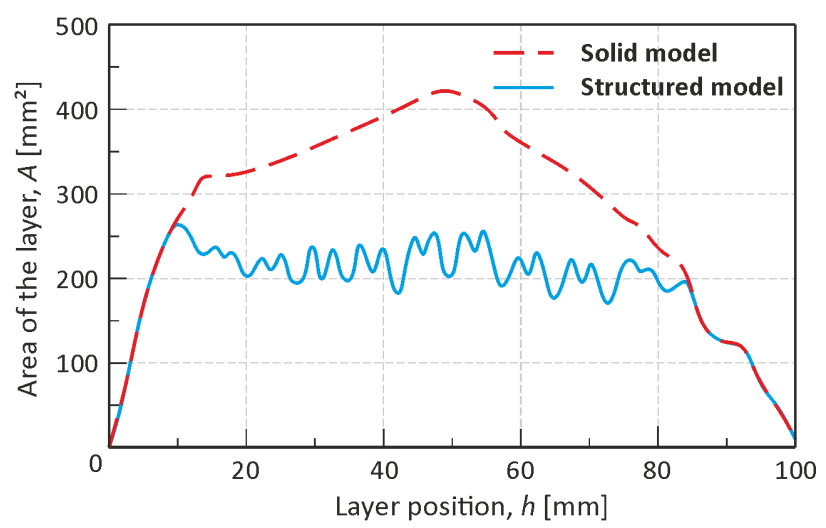

Figure 5. Area of individual layers throughout the solid and the structured model.

\subsection{Fabrication of the samples and post-process operations}

All the previously described specimens were fabricated during one build job using the SLM280HL machine (SLM Solutions AG, Lübeck, Germany). The job was built with $30 \mu \mathrm{m}$ layer thickness and under Argon protecting atmosphere. For core part of the model, laser power of $275 \mathrm{~W}$, scanning speed of $1100 \mathrm{~mm} / \mathrm{s}$. and hatch distance between neighbouring laser paths of $0.12 \mathrm{~mm}$ were used. Such combination of parameters gives volume energy density of $E=76.4 \mathrm{~J} / \mathrm{mm}^{3}$. Output of the process is a structure with minimal porosity. 


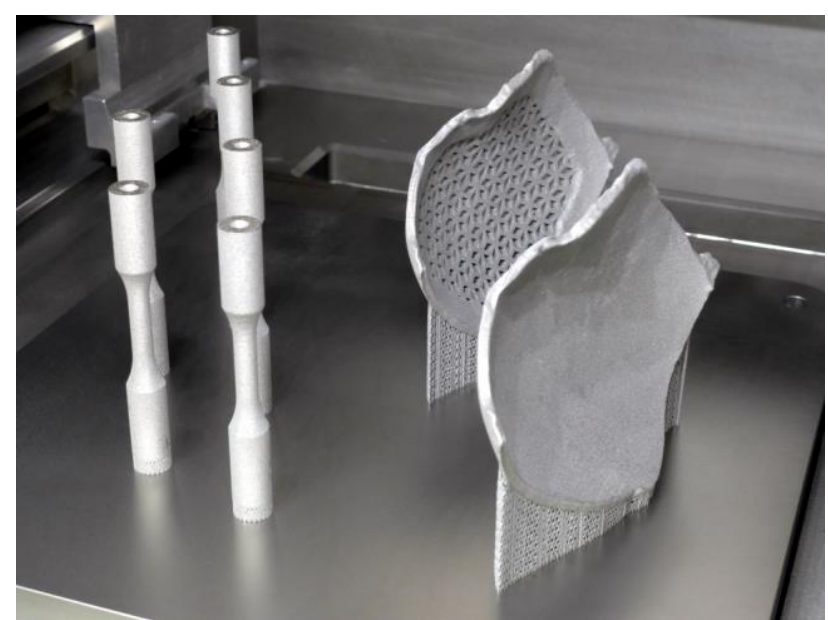

Figure 6. Tensile test specimens and cranial implants after fabrication.

Post processing part of the fabrication contained following steps: Three of the six tensile test specimens were removed from substrate plate right after the fabrication - these samples were tested in as-built condition. Rest of the specimens underwent stress relief heat treatment which consisted of $50^{\circ} \mathrm{C} / \mathrm{min}$ ramp heating to $640^{\circ} \mathrm{C}, 4 \mathrm{~h}$ annealing at the temperature and slow cooling in the oven.

Tensile test specimens were finished using conventional turning operations according to drawing in the fig. 3 . In the case of cranial implants, support structures were removed and the products were submitted to finishing operations to gain polished surface quality.

\subsection{Tensile testing}

After finishing of the samples, tensile test of the six specimens was done on universal testing machine TIRA Test equipped with load cell having $\pm 100 \mathrm{kN}$ loading capacity. Each test started with $1 \mathrm{kN}$ pretension after which clip-on extensometer EPSILON 3542 was fixed to the sample (Fig. 7). Initial length between extensometer's blades was $10 \mathrm{~mm}$. The test was carried out until rupture of the specimen.

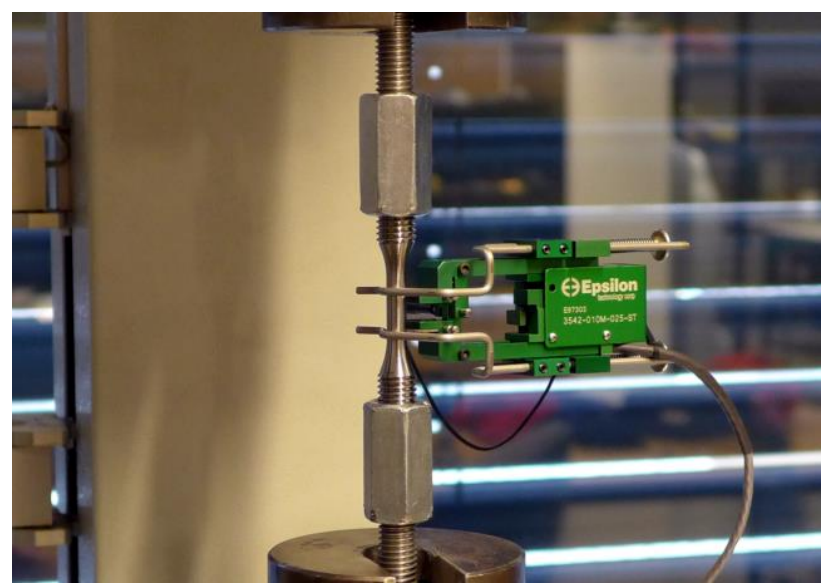

Figure 7. Experimental setup of the tensile test.

\subsection{Shape accuracy measurement}

Both versions of cranial implant were digitized using GOM ATOS II optical 3D scanning device. In the first step, thin film of chalk was deposited to the surface of the part in order to reduce reflections and possible noise in the measurement. Scanned part was placed to rotating plate on which 10 individual images were acquired (Fig. 8).

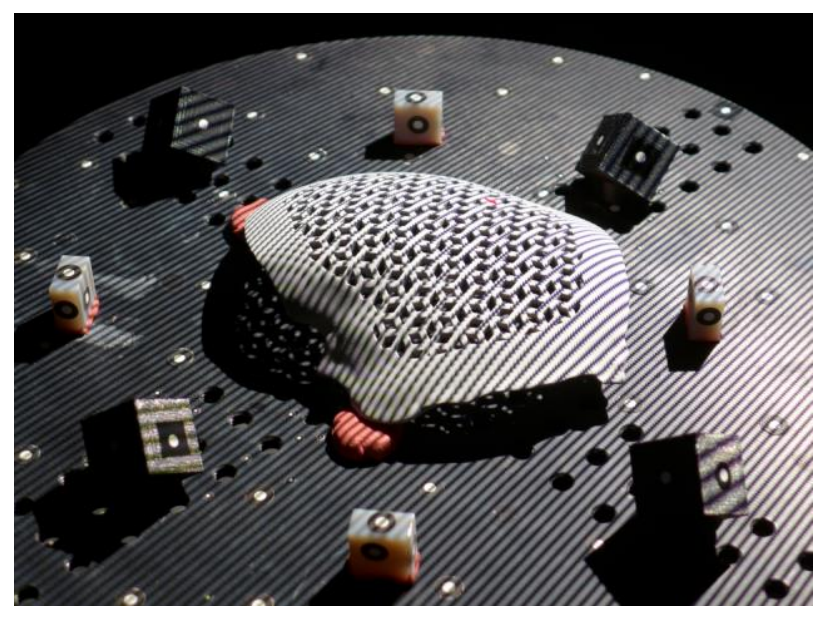

Figure 8. Shape accuracy measurement (structured part).

Digitization and comparison with original CAD data was done in GOM Inspect evaluation software. As a result, colour map of normal deflections from nominal shape was calculated.

\section{RESULTS}

\subsection{Tensile tests}

In the fig. 9, stress-strain diagrams of tensile test are shown for individual tested specimens. For both groups of specimens heat treated and as-built, similar behaviour of the tensile test curve can be seen. The alloy exhibit distinct linear stress-strain dependency in the initial part of the diagram followed by almost immediate transition to the plastic state.

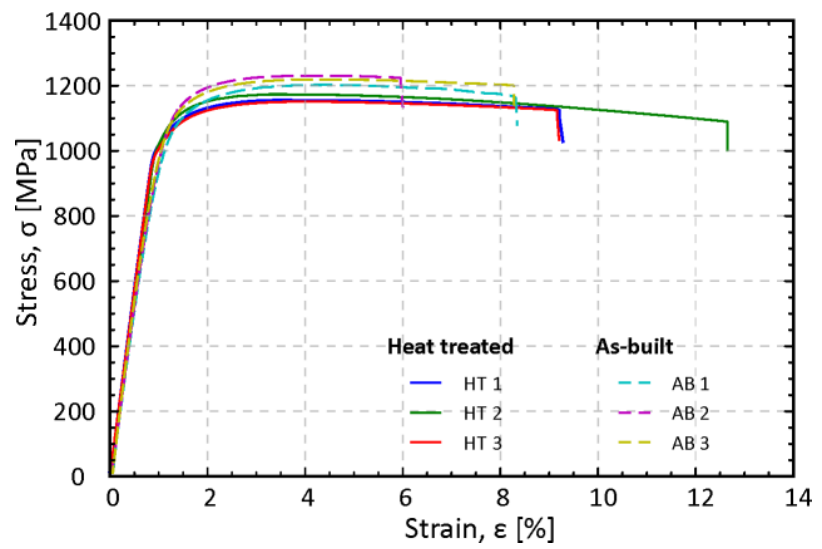

Figure 9. Tensile test diagram of SLM-processed Ti-6AI-4V alloy

On the basis of tensile test data, standard mechanical properties were calculated - specifically, Offset yield stress $R_{p 0,2}$, Ultimate tensile stress $R_{m}$, Elongation at break $\varepsilon_{b}$ and Young's modulus E (Tab. 1). As apparent form the diagrams, heat treatment of the alloy after the SLM process increases strain capabilities of the material with low reduction of Ultimate tensile stress.

For conventional Ti-6Al-4V material, following mechanical properties can be found: $R_{p 0,2}=850-900 \mathrm{MPa}, R_{m}=960-$ $970 \mathrm{MPa}$ and $\mathrm{E}=110000 \mathrm{MPa}$ [Ratner 2004]. In comparison with these data, it is clear that SLM technology produces more brittle structure with higher stress thresholds. This fact should not, on the other hand, represent any drawback in using the technology for this purpose. 


\begin{tabular}{|c|c|c|c|c|}
\hline & $\begin{array}{c}\mathbf{R}_{\mathbf{p}, 2} \\
{[\mathrm{MPa}]}\end{array}$ & $\begin{array}{c}\mathbf{R m} \\
{[\mathrm{MPa}]}\end{array}$ & $\begin{array}{c}\varepsilon_{\mathrm{b}} \\
{[\%]}\end{array}$ & $\begin{array}{c}\mathbf{E} \\
{[\mathrm{MPa}]}\end{array}$ \\
\hline $\mathrm{HT} 1$ & 1044 & 1156 & 8.38 & 114344 \\
\hline $\mathrm{HT2}$ & 1067 & 1174 & 11.74 & 109904 \\
\hline $\mathrm{HT3}$ & 1030 & 1152 & 8.29 & 114144 \\
\hline AB1 & 1044 & 1202 & 7.27 & 100823 \\
\hline AB2 & 1089 & 1232 & 4.92 & 103383 \\
\hline AB3 & 1070 & 1220 & 7.21 & 106334 \\
\hline
\end{tabular}

Table 1. Standard mechanical constants for tested Ti-6Al-4V specimens

\subsection{Shape accuracy}

Fig. 10 shows both solid and lightweight version of tested cranial implant with colour map of nominal deviations versus the nominal shape. In general, both geometries were found to have large deflections at sharp ends of the geometry. Main reason for this fact is that these areas were unsupported.

Another finding which can be derived from the data is that even with the same supporting technique and building orientation, the structured model evince better conformity with original data. In comparison with the area with largest deviation of solid model $(-2.02 \mathrm{~mm})$, the deviation in the same place is $-1.11 \mathrm{~mm}$ for structured model. This finding is similar to all the observed areas of the structured part.
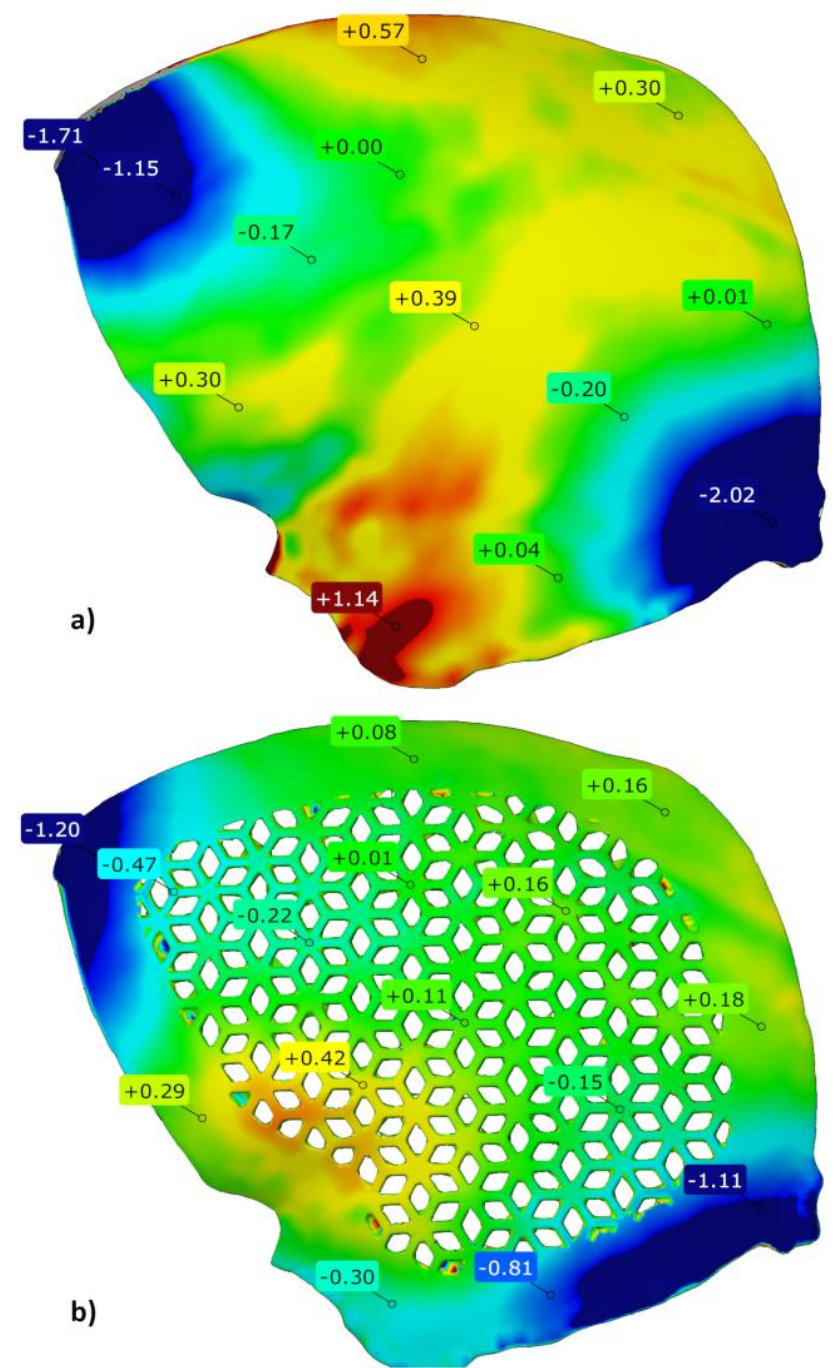

Figure 10. Shape accuracy analysis of a) solid and b) lightweight cranial implant

\section{CONCLUSIONS}

In this work, the biocompatible Ti-6Al-4V alloy and its use for Selective Laser Melting additive technology was studied. Main focus was paid to two aspects of the final product - mechanical properties of the material and shape accuracy of the fabrication process.

In the first part, the work dealt with evaluation of mechanical properties in as-built condition and in the state after stress relief heat treatment. In comparison with available data for the bulk material, SLM process produces more brittle structure with higher value of Ultimate tensile stress and lower value of strain at break. Although the stress relief heat treatment bring these values closer to the mechanical constants of a conventional alloy, structure still remains considerably stiffer. With respect to intended use of SLM-processed Ti-6AI-4V alloy for individual implants, this state would not mean any drawback for use of the method.

Second part of the work was aimed to shape accuracy study of a sample part in the form of a cranial implant. Additive technologies are known for a possibility of building very complex structures. Thus, two versions of the implant were compared. On one side, the shape was left in the condition typical for standard milling operations - curved solid plate. On the second side, lightweight structure was incorporated to the geometry. 3D scanning of the two models after fabrication showed that unsupported parts of both implants were considerably distorted. Thus, more attention should be paid to data preparation of the build job. Main finding of this work was that implementation of the structures into a model not only reduces build time of job but it also lowers deflections due to smaller scanned area. It can be stated that proper use of structures is one of the very promising features for use in the field of individual implants.

Future work will deal with simulation of the build job and solving the deflection problems and its possible reduction in pre-processing part. Moreover, various structures will be studied for further use in SLM process.

\section{ACKNOWLEDGMENTS}

The research presented in this article was supported by the Ministry of Education, Youth and Sports in the framework of the targeted support of the OPR \& DI project "Extension of CxI facilities" (CZ.1.05/2.1.00/19.0386).

\section{REFERENCES}

[Eufinger 1995] Eufinger, H., et al. (1995) Reconstruction of craniofacial bone defects with individual alloplastic implants based on CAD/CAM-manipulated CT-data. Journal of Cranio-Maxillofacial Surgery 23.3: 175181.

[Hao 2016] Hao, Y.-L., Shu-J. L., and Rui Y. (2016) Biomedical titanium alloys and their additive manufacturing. Rare Metals 35.9: 661-671.

[Murr 2012] Murr, L. E. et al. (2012). Metal fabrication by additive manufacturing using laser and electron beam melting technologies. Journal of Materials Science \& Technology, 28(1), 1-14. 
[Ratner 2004] Ratner, B. D., Hoffman, A. S., Schoen, F. J., \& Lemons, J. E. (2004). Biomaterials science: an introduction to materials in medicine. Elsevier. ISBN 9780123746269
[Sing 2016] Sing, S. L. et al. (2016). Laser and electron-beam powder-bed additive manufacturing of metallic implants: A review on processes, materials and designs. Journal of Orthopaedic Research, 34(3), 369-385.

\section{CONTACTS}

Ing. Michal Ackermann, Ph.D.

Technical University of Liberec, Institute for Nanomaterials, Advanced Technologies and Innovation.

Studentska 1402/2, Liberec, 461 17, Czech Republic

+420 485353 723, michal.ackermann@tul.cz 